Received 5 February 2016

Accepted 8 February 2016

Edited by W. T. A. Harrison, University of Aberdeen, Scotland

Keywords: crystal structure; 2,6-diaminopyridinium cation; hybrid salt; hydrogen bonding.

CCDC reference: 1452262

Supporting information: this article has supporting information at journals.iucr.org/e

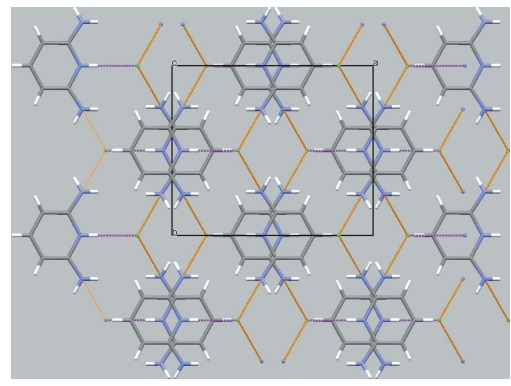

OPEN $\odot$ ACCESS

\section{Crystal structure of 2,6-diaminopyridinium chloride}

\author{
Matthias Mastalir, ${ }^{a}$ Martina Schroffenegger, ${ }^{a}$ Berthold Stöger, ${ }^{b}$ Matthias Weil ${ }^{\text {b* }}$ \\ and Karl Kirchner ${ }^{\mathrm{a}}$
}

\begin{abstract}
anstitute of Applied Synthetic Chemistry, TU Wien, Getreidemarkt 9/163, A-1060 Vienna, Austria, and ${ }^{\mathbf{b}}$ Institute for Chemical Technologies and Analytics, Division of Structural Chemistry, TU Wien, Getreidemarkt 9/164-SC, A-1060 Vienna, Austria. *Correspondence e-mail: Matthias.Weil@tuwien.ac.at
\end{abstract}

The asymmetric unit of the title salt, $\mathrm{C}_{5} \mathrm{H}_{8} \mathrm{~N}_{3}{ }^{+} \cdot \mathrm{Cl}^{-}$, comprises one half of the 2,6diaminopyridinium cation (the other half being completed by the application of mirror symmetry) and one $\mathrm{Cl}^{-}$counter-anion, also located on the mirror plane. The amino $\mathrm{N}$ atom shows a significant pyramidalization, with a dihedral angle of $30.4(14)^{\circ}$ between the least-squares planes of the amino group and the non- $\mathrm{H}$ atoms of the 2,6-diaminopyridinium moiety. In the crystal, the cationic molecules and $\mathrm{Cl}^{-}$counter-anions are arranged in sheets parallel to (001) consisting of alternating polar and non-polar parts associated with the the $\mathrm{Cl}^{-}$ anions, pyridinium and amino moieties, and the pyridine rings, respectively. $\mathrm{N}-$ $\mathrm{H}$. C Cl interactions within the polar part, as well as slipped $\pi-\pi$ interactions in the non-polar part, help to establish the three-dimensional network.

\section{Chemical context}

Pincer compounds are an important class of chelating ligands, and their metal complexes have attracted tremendous interest due to their high stability, activity, variability and applicability in organic synthesis and catalysis (Szabo \& Wendt, 2014). Whereas a plethora of (mostly) precious transition-metal pincer complexes has been reported, information on group 6 pincer complexes is rather scarce. During a project aimed at the preparation and characterization of group 6 PNP pincer compounds (Öztopcu et al., 2013; de Aguiar et al., 2014; Mastalir et al., 2016), crystals of the title salt, $\mathrm{C}_{5} \mathrm{H}_{8} \mathrm{~N}_{3}{ }^{+} \cdot \mathrm{Cl}^{-}$, were obtained accidentally through hydrolysis of the employed ligand $N, N^{\prime}$-bis(diisopropylphosphino)-2,6-diaminopyridine in the presence of $\mathrm{CrCl}_{3} \cdot 6 \mathrm{H}_{2} \mathrm{O}$. Here we report on the crystal structure of this salt.

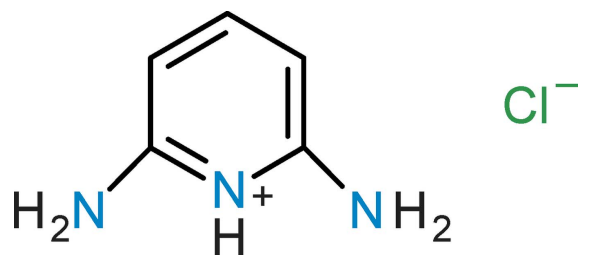

\section{Structural commentary}

The cation of the title structure is protonated at the pyridine $\mathrm{N}$ atom (Fig. 1). The asymmetric unit comprises half a molecule of the 2,6-diaminopyridinium cation, with a mirror plane running through the pyridinium group $(\mathrm{N} 1-\mathrm{H} 1 \mathrm{~N} 1)$ and the para-C $-\mathrm{H}$ group $(\mathrm{C} 3-\mathrm{H} 1 \mathrm{C} 3)$; the $\mathrm{Cl}^{-}$anion is also located on the mirror plane. In agreement with other 2,6-diamino- 


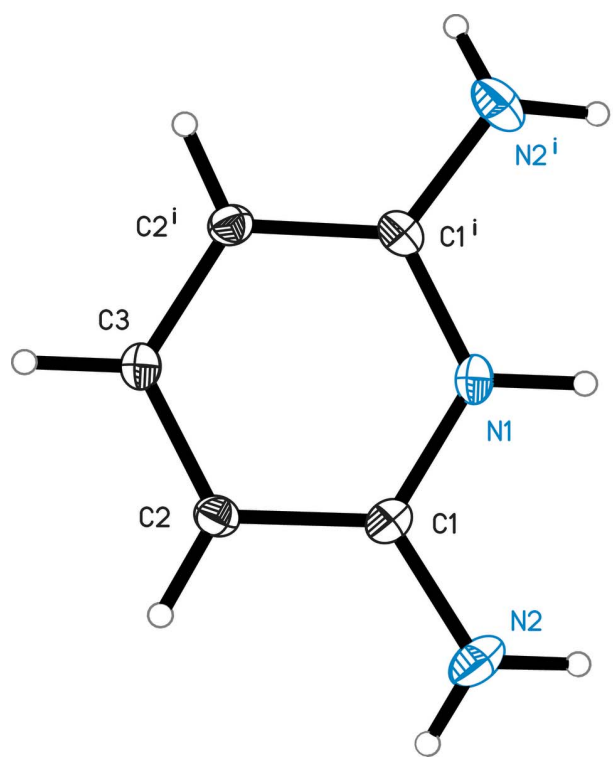

Figure 1

The molecular structure of the cation and the inorganic anion in the title structure. Displacement ellipsoids are drawn at the $50 \%$ probability level. [Symmetry code: (i) $x,-y, z$.]

pyridinium cations, the $\mathrm{C}-\mathrm{N}(\mathrm{H})^{+}-\mathrm{C}$ angle involving the pyridinium group is enlarged $\left[\mathrm{C} 1-\mathrm{N} 1-\mathrm{C}^{\mathrm{i}}=123.37(8)^{\circ}\right.$; symmetry code: (i) $x,-y, z]$ whereas the angle between the pyridinium $\mathrm{N}$ atom and the $\mathrm{C}$ atom in the ortho position (bearing the amino group) and in the meta position is reduced $\left[\mathrm{N} 1-\mathrm{C} 1-\mathrm{C} 2=118.83(6)^{\circ}\right]$. This situation is reversed in 2,6diaminopyridine due to the non-protonated ring $\mathrm{N}$ atom in this structure (Schwalbe et al., 1987). A common feature of the non-protonated 2,6-diaminopyridine molecule and the 2,6diaminopyridinium cation is a significant pyramidalization of the amino $\mathrm{N}$ atom. In the title structure, the bond angle sum at this atom (N2) deviates with $349.0^{\circ}$ clearly from the expected

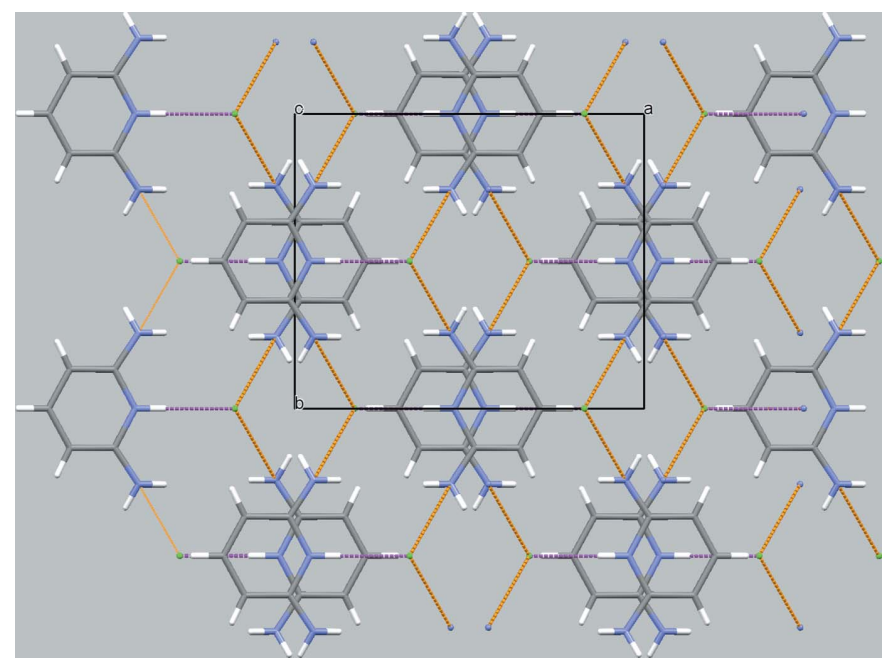

Figure 2

Crystal packing of the organic and inorganic components in the title structure in a projection along [001]. $\mathrm{N}-\mathrm{H} \cdots \mathrm{Cl}$ hydrogen bonds involving the pyridinium group are shown as magenta dotted lines and those involving the amino group are shown as orange dotted lines.
Table 1

Hydrogen-bond geometry $\left(\AA{ }^{\circ}\right)$.

\begin{tabular}{lllll}
\hline$D-\mathrm{H} \cdots A$ & $D-\mathrm{H}$ & $\mathrm{H} \cdots A$ & $D \cdots A$ & $D-\mathrm{H} \cdots A$ \\
\hline $\mathrm{N} 1-\mathrm{H} 1 N 1 \cdots \mathrm{Cl} 1$ & $0.90(2)$ & $2.18(2)$ & $3.0790(11)$ & $175.6(19)$ \\
$\mathrm{N} 2-\mathrm{H} 2 N 2 \cdots \mathrm{Cl} 1^{\mathrm{i}}$ & $0.833(13)$ & $2.628(13)$ & $3.4086(8)$ & $156.5(12)$ \\
$\mathrm{N} 2-\mathrm{H} 1 N 2 \cdots \mathrm{Cl} 1^{\mathrm{ii}}$ & $0.875(13)$ & $2.877(13)$ & $3.3601(8)$ & $116.8(2)$ \\
\hline
\end{tabular}

Symmetry codes: (i) $x+\frac{1}{2}, y+\frac{1}{2}, z$; (ii) $-x+\frac{1}{2},-y+\frac{1}{2},-z$.

$360^{\circ}$ for an ideal trigonal-planar group. The pyramidalization is also reflected by the dihedral angle of $30.4(14)^{\circ}$ between the least-squares planes of the amino group and the non-H atoms of the 2,6-diaminopyridinium moiety.

\section{Supramolecular features}

The pyridinium $\mathrm{N} 1-\mathrm{H} 1 \mathrm{~N} 1$ group is the donor of a nearly linear hydrogen bond to the $\mathrm{Cl}^{-}$counter anion (Table 1). The amino group also participates in the formation of $\mathrm{N}-\mathrm{H} \cdots \mathrm{Cl}$ hydrogen bonds, albeit of explicit weaker nature. One hydrogen atom $(\mathrm{H} 2 \mathrm{~N} 2)$ is clearly involved in hydrogen bonding with an $\mathrm{H} 2 \mathrm{~N} 2 \cdots \mathrm{Cl} 1$ distance of $2.63 \AA$ and an $\mathrm{N} 2-$ $\mathrm{H} 2 \mathrm{~N} 2 \cdots \mathrm{Cl} 1$ angle of $157^{\circ}$. Although the $D \cdots A$ contact involving the second hydrogen atom, $\mathrm{H} 2 \mathrm{~N} 2$, is $0.04 \AA$ shorter than that of the other hydrogen bond of this group, the comparatively long $\mathrm{H} 1 \mathrm{~N} 2 \cdots \mathrm{Cl}$ distance of $2.88 \AA$ and the very small $\mathrm{N} 2-\mathrm{H} 1 N 2 \cdots \mathrm{Cl} 1$ angle of $117^{\circ}$ give room for interpretation whether or not this is a real hydrogen bond.

In the crystal (Figs. 2 and 3), the cationic molecules and anions are arranged into layers with alternating polar and nonpolar parts extending parallel to (001). Adjacent polar parts, comprising the $\mathrm{Cl}^{-}$anions and the pyridinium and amino moieties, are linked through $\mathrm{N}-\mathrm{H} \cdot \mathrm{Cl}$ hydrogen bonds into sheets with a thickness of $\simeq c / 2$. The non-polar parts, i.e. the pyridine rings, interact through slipped $\pi-\pi$ stacking along [001] with a centroid-to-centroid distance of 3.5129 (6) $\AA$; the corresponding plane-to-plane distance between the pyridine rings is $3.344 \AA$.

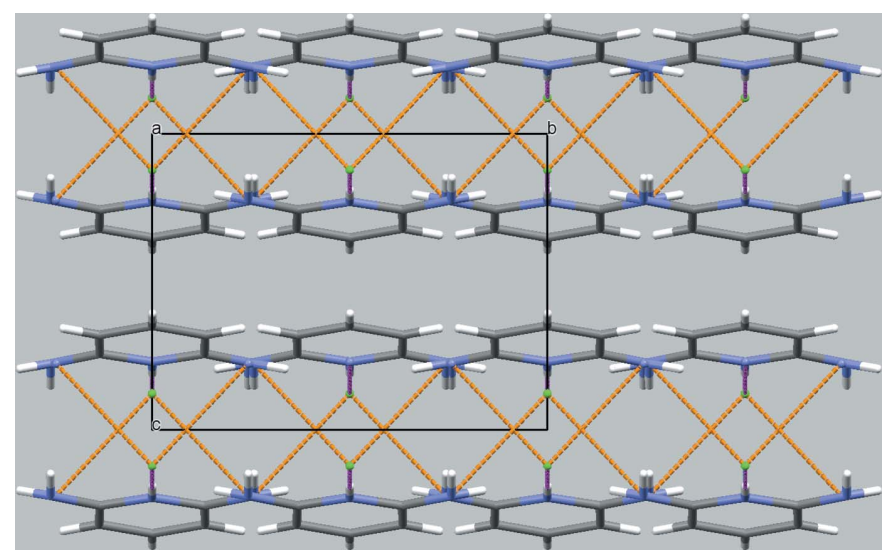

Figure 3

Crystal packing of the organic and inorganic components in the title structure in a projection along [100]. The colour code of the intermolecular interactions is as in Fig. 2. 
Table 2

Experimental details.

\begin{tabular}{|c|c|}
\hline \multicolumn{2}{|l|}{ Crystal data } \\
\hline Chemical formula & $\mathrm{C}_{5} \mathrm{H}_{8} \mathrm{~N}^{3+} \cdot \mathrm{Cl}^{-}$ \\
\hline$M_{\mathrm{r}}$ & 145.6 \\
\hline Crystal system, space group & Monoclinic, $C 2 / m$ \\
\hline Temperature $(\mathrm{K})$ & 100 \\
\hline$a, b, c(\AA)$ & $10.8046(10), 9.0459(9), 6.8108(7)$ \\
\hline$\beta\left(^{\circ}\right)$ & $96.710(2)$ \\
\hline$V\left(\AA^{3}\right)$ & $661.11(11)$ \\
\hline$Z$ & 4 \\
\hline Radiation type & Мо $K \alpha$ \\
\hline$\mu\left(\mathrm{mm}^{-1}\right)$ & 0.48 \\
\hline Crystal size (mm) & $0.52 \times 0.38 \times 0.23$ \\
\hline \multicolumn{2}{|l|}{ Data collection } \\
\hline Diffractometer & Bruker Kappa APEXII CCD \\
\hline Absorption correction & $\begin{array}{l}\text { Multi-scan (SADABS; Bruker, } \\
\text { 2014) }\end{array}$ \\
\hline$T_{\min }, T_{\max }$ & $0.80,0.90$ \\
\hline $\begin{array}{l}\text { No. of measured, independent and } \\
\text { observed }[I>3 \sigma(I)] \text { reflections }\end{array}$ & $9529,1538,1407$ \\
\hline$R_{\text {int }}$ & 0.031 \\
\hline$(\sin \theta / \lambda)_{\max }\left(\AA^{-1}\right)$ & 0.808 \\
\hline \multicolumn{2}{|l|}{ Refinement } \\
\hline$R\left[F^{2}>3 \sigma(F)\right], w R(F), S$ & $0.024,0.038,2.24$ \\
\hline No. of reflections & 1538 \\
\hline No. of parameters & 64 \\
\hline $\mathrm{H}$-atom treatment & All H-atom parameters refined \\
\hline$\Delta \rho_{\max }, \Delta \rho_{\min }\left(\mathrm{e} \AA^{-3}\right)$ & $0.49,-0.18$ \\
\hline
\end{tabular}

Computer programs: APEX2 (Bruker, 2014), SAINT (Bruker, 2014), SUPERFLIP (Palatinus \& Chapuis, 2007), JANA2006 (Petříček et al., 2014), XP in SHELXTL (Sheldrick, 2008), Mercury (Macrae et al., 2006) and publCIF (Westrip, 2010).

\section{Database survey}

A search in the CSD (Groom \& Allen, 2014; CSD Version 5.31) revealed 87 different salts containing the 2,6-diaminopyridinium cation, with the majority of cases in the form of organic anions (46 representatives), followed by complex metal anions (31 representatives). Two structures are reported that contain additional metal cations and inorganic anions, and eight representatives are compiled with inorganic anions only, including the $\mathrm{SiF}_{6}{ }^{2-}$ salt (CSD code FOSXER; Gelmboldt et al., 2009), the $\mathrm{Br}^{-}$salt (GOLMIF; Turrell et al., 2010), the $\mathrm{BF}_{4}{ }^{-}$salt (IFOQAW; Benito-Garagorri et al.; 2007), the $\mathrm{Br}^{-}$ salt monohydrate (ILINEW; Haddad \& Al-Far, 2003), the hydrogensulfate sulfate salt (KORRAM; Said \& Naili, 2014), the $\mathrm{ClO}_{4}{ }^{-}$salt (MIGWOP; Jazdon et al., 2007), the $\mathrm{H}_{2} \mathrm{PO}_{4}{ }^{-}$ salt (QEDHUE; Yu, 2012) and the $\mathrm{NO}_{3}{ }^{-}$salt (XAKVAG; Kristiansson, 2000). It should be noted that the chemically most related anhydrous $\mathrm{Br}^{-}$salt crystallizes in space group $I \overline{4} 2 d$ and hence shows no isotypism with the title $\mathrm{Cl}^{-}$salt.

\section{Synthesis and crystallization}

$N, N$ '-bis(diisopropylphosphino)-2,6-diaminopyridine $\quad(0.2 \mathrm{~g}$, $0.53 \mathrm{mmol}$ ) was dissolved in dry tetrahydrofuran $(5 \mathrm{ml})$ under argon atmosphere. $\mathrm{CrCl}_{3} \cdot 6 \mathrm{H}_{2} \mathrm{O}(0.134 \mathrm{~g}, 0.51 \mathrm{mmol})$ was added and the resulting mixture stirred for $4 \mathrm{~h}$ at room temperature. The formed purple solid was filtered off, washed with dry diethyl ether and dried. The solid was redissolved in acetonitrile for crystallization initiated by solvent diffusion with diethyl ether. The title compound grew in the form of yellow crystals as the only solid product. We assume that the Lewis acid $\mathrm{CrCl}_{3}$ in combination with water is able to cleave the $\mathrm{P}-\mathrm{N}$ bond of the pincer compound accompanied by an in situ formation of $\mathrm{HCl}$ which eventually yields the title compound.

\section{Refinement}

All $\mathrm{H}$ atoms were clearly discernible from difference Fourier maps and were refined freely. Crystal data, data collection and structure refinement details are summarized in Table 2.

\section{Acknowledgements}

The X-ray centre of TU Wien is acknowledged for providing access to the single-crystal diffractometer.

\section{References}

Aguiar, S. R. M. M. de, Öztopcu, Ö., Stöger, B., Mereiter, K., Veiros, L. F., Pittenauer, E., Allmaier, G. \& Kirchner, K. (2014). Dalton Trans. 43, 14669-14679.

Benito-Garagorri, D., Kirchner, K. \& Mereiter, K. (2007). Private communication (refcode IFOQAW). CCDC, Cambridge, England.

Bruker (2014). APEX2, SAINT and SADABS. Bruker AXS Inc., Madison, Wisconsin, USA.

Gelmboldt, V. O., Ganin, E. V., Fonari, M. S., Koroeva, L. V., Ivanov, Y. E. \& Botoshansky, M. M. (2009). J. Fluor. Chem. 130, 428-433. Groom, C. R. \& Allen, F. H. (2014). Angew. Chem. Int. Ed. 53, 662671.

Haddad, S. F. \& Al-Far, R. H. (2003). Acta Cryst. E59, o1444-o1446. Jazdoń, M., Radecka-Paryzek, W. \& Kubicki, M. (2007). Acta Cryst. E63, o3337.

Kristiansson, O. (2000). Z. Kristallogr. New Cryst. Struct. 215, 138.

Macrae, C. F., Edgington, P. R., McCabe, P., Pidcock, E., Shields, G. P., Taylor, R., Towler, M. \& van de Streek, J. (2006). J. Appl. Cryst. 39, 453-457.

Mastalir, M., de Aguiar, S. R. M. M., Glatz, M., Stöger, B. \& Kirchner, K. (2016). Organometallics, 35, 229-232.

Öztopcu, Ö., Holzhacker, C., Puchberger, M., Weil, M., Mereiter, K., Veiros, L. F. \& Kirchner, K. (2013). Organometallics, 32, 3042-3052. Palatinus, L. \& Chapuis, G. (2007). J. Appl. Cryst. 40, 786-790.

Petř́ček, V., Dušek, M. \& Palatinus, L. (2014). Z. Kristallogr. 229, $345-352$.

Said, S. \& Naili, H. (2014). Private communication (refcode KORRAM). CCDC, Cambridge, England.

Schwalbe, C. H., Williams, G. J. B. \& Koetzle, T. F. (1987). Acta Cryst. C43, 2191-2195.

Sheldrick, G. M. (2008). Acta Cryst. A64, 112-122.

Szabo, K. J. \& Wendt, O. F. (2014). In Pincer and Pincer-Type Complexes: Applications in Organic Synthesis and Catalysis. Weinheim: Wiley-VCH.

Turrell, P. J., Wright, J. A. \& Pickett, C. J. (2010). Private communication (refcode GOLMIF). CCDC, Cambridge, England.

Westrip, S. P. (2010). J. Appl. Cryst. 43, 920-925.

Yu, G. (2012). Acta Cryst. E68, o2751. 


\section{supporting information}

Acta Cryst. (2016). E72, 331-333 [doi:10.1107/S2056989016002425]

Crystal structure of 2,6-diaminopyridinium chloride

Matthias Mastalir, Martina Schroffenegger, Berthold Stöger, Matthias Weil and Karl Kirchner

Computing details

Data collection: APEX2 (Bruker, 2014); cell refinement: SAINT (Bruker, 2014); data reduction: SAINT (Bruker, 2014); program(s) used to solve structure: SUPERFLIP (Palatinus \& Chapuis, 2007); program(s) used to refine structure:

JANA2006 (Petrríček et al., 2014); molecular graphics: XP in SHELXTL (Sheldrick, 2008) and Mercury (Macrae et al., 2006); software used to prepare material for publication: publCIF (Westrip, 2010).

2,6-Diaminopyridin-1-ium chloride

Crystal data

$\mathrm{C}_{5} \mathrm{H}_{8} \mathrm{~N}^{3+} \cdot \mathrm{Cl}^{-}$

$M_{r}=145.6$

Monoclinic, $C 2 / m$

Hall symbol: $-\mathrm{C} 2 \mathrm{y}$

$a=10.8046(10) \AA$

$b=9.0459(9) \AA$

$c=6.8108(7) \AA$

$\beta=96.710(2)^{\circ}$

$V=661.11(11) \AA^{3}$

$Z=4$

\section{Data collection}

Bruker Kappa APEXII CCD diffractometer

Radiation source: X-ray tube Graphite monochromator

$\omega$ - and $\varphi$-scans

Absorption correction: multi-scan

(SADABS; Bruker, 2014)

$T_{\text {min }}=0.80, T_{\max }=0.90$

Refinement

Refinement on $F$

$R\left[F^{2}>2 \sigma\left(F^{2}\right)\right]=0.024$

$w R\left(F^{2}\right)=0.038$

$S=2.24$

1538 reflections

64 parameters

0 restraints
$F(000)=304$

$D_{\mathrm{x}}=1.462 \mathrm{Mg} \mathrm{m}^{-3}$

Mo $K \alpha$ radiation, $\lambda=0.71073 \AA$

Cell parameters from 6316 reflections

$\theta=2.9-35.5^{\circ}$

$\mu=0.48 \mathrm{~mm}^{-1}$

$T=100 \mathrm{~K}$

Block, yellow

$0.52 \times 0.38 \times 0.23 \mathrm{~mm}$

9529 measured reflections

1538 independent reflections

1407 reflections with $I>3 \sigma(I)$

$R_{\text {int }}=0.031$

$\theta_{\text {max }}=35.0^{\circ}, \theta_{\min }=3.0^{\circ}$

$h=-17 \rightarrow 17$

$k=-14 \rightarrow 14$

$l=-10 \rightarrow 10$

0 constraints

All H-atom parameters refined

Weighting scheme based on measured s.u.'s $w=$

$1 /\left(\sigma^{2}(F)+0.0001 F^{2}\right)$

$(\Delta / \sigma)_{\max }=0.030$

$\Delta \rho_{\max }=0.49$ e $\AA^{-3}$

$\Delta \rho_{\min }=-0.18$ e $\AA^{-3}$ 
Fractional atomic coordinates and isotropic or equivalent isotropic displacement parameters $\left(\AA^{2}\right)$

\begin{tabular}{lllll}
\hline & $x$ & $y$ & $z$ & $U_{\text {iso }} * / U_{\text {eq }}$ \\
\hline C11 & $0.17115(2)$ & 0 & $0.12206(3)$ & $0.01401(7)$ \\
$\mathrm{N} 1$ & $0.45600(9)$ & 0 & $0.22925(12)$ & $0.0132(2)$ \\
$\mathrm{N} 2$ & $0.44617(7)$ & $0.25682(8)$ & $0.22357(10)$ & $0.01951(18)$ \\
$\mathrm{C} 1$ & $0.51532(7)$ & $0.13257(7)$ & $0.25660(9)$ & $0.01272(16)$ \\
$\mathrm{C} 2$ & $0.64225(7)$ & $0.13396(7)$ & $0.32011(10)$ & $0.01334(17)$ \\
$\mathrm{C} 3$ & $0.70393(10)$ & 0 & $0.35111(14)$ & $0.0136(2)$ \\
$\mathrm{H} 1 \mathrm{C} 2$ & $0.6796(9)$ & $0.2256(14)$ & $0.3421(16)$ & $0.019(3)^{*}$ \\
$\mathrm{H} 1 \mathrm{C} 3$ & $0.7905(16)$ & 0 & $0.392(2)$ & $0.015(3)^{*}$ \\
$\mathrm{H} 1 \mathrm{~N} 2$ & $0.3742(16)$ & $0.2574(17)$ & $0.150(2)$ & $0.046(4)^{*}$ \\
$\mathrm{H} 2 \mathrm{~N} 2$ & $0.4841(12)$ & $0.3350(15)$ & $0.2058(19)$ & $0.032(3)^{*}$ \\
$\mathrm{H} 1 \mathrm{~N} 1$ & $0.3735(19)$ & 0 & $0.191(3)$ & $0.039(5)^{*}$ \\
\end{tabular}

Atomic displacement parameters $\left(\AA^{2}\right)$

\begin{tabular}{lllllll}
\hline & $U^{11}$ & $U^{22}$ & $U^{33}$ & $U^{12}$ & $U^{13}$ & $U^{23}$ \\
\hline C11 & $0.01121(13)$ & $0.01270(11)$ & $0.01763(12)$ & 0 & $-0.00037(8)$ & 0 \\
N1 & $0.0093(4)$ & $0.0176(4)$ & $0.0128(3)$ & 0 & $0.0018(3)$ & 0 \\
N2 & $0.0192(3)$ & $0.0187(3)$ & $0.0214(3)$ & $0.0075(2)$ & $0.0056(2)$ & $0.0065(2)$ \\
C1 & $0.0137(3)$ & $0.0143(3)$ & $0.0107(2)$ & $0.0025(2)$ & $0.0039(2)$ & $0.00180(19)$ \\
C2 & $0.0136(3)$ & $0.0118(3)$ & $0.0149(3)$ & $-0.0012(2)$ & $0.0026(2)$ & $-0.00027(19)$ \\
C3 & $0.0108(4)$ & $0.0154(4)$ & $0.0145(4)$ & 0 & $0.0011(3)$ & 0 \\
\hline
\end{tabular}

Geometric parameters $(\AA, \stackrel{\circ}{)})$

\begin{tabular}{llll}
\hline $\mathrm{N} 1-\mathrm{C} 1$ & $1.3622(8)$ & $\mathrm{C} 1-\mathrm{C} 2$ & $1.3890(10)$ \\
$\mathrm{N} 1-\mathrm{C} 1$ & $1.3622(8)$ & $\mathrm{C} 2-\mathrm{C} 3$ & $1.3872(9)$ \\
$\mathrm{N} 1-\mathrm{H} 1 \mathrm{~N} 1$ & $0.90(2)$ & $\mathrm{C} 2-\mathrm{H} 1 \mathrm{C} 2$ & $0.927(12)$ \\
$\mathrm{N} 2-\mathrm{C} 1$ & $1.3538(10)$ & $\mathrm{C} 3-\mathrm{H} 1 \mathrm{C} 3$ & $0.944(17)$ \\
$\mathrm{N} 2-\mathrm{H} 1 \mathrm{~N} 2$ & $0.875(16)$ & $\mathrm{C} 3-\mathrm{C} 2$ & $1.3872(9)$ \\
$\mathrm{N} 2-\mathrm{H} 2 \mathrm{~N} 2$ & $0.833(13)$ & & \\
& & & $123.35(6)$ \\
$\mathrm{C} 1-\mathrm{N} 1-\mathrm{C} 1$ & $123.37(8)$ & $\mathrm{N} 2-\mathrm{C} 1-\mathrm{C} 2$ & $118.60(7)$ \\
$\mathrm{C} 1-\mathrm{N} 1-\mathrm{H} 1 \mathrm{~N} 1$ & $118.32(4)$ & $\mathrm{C} 1-\mathrm{C} 2-\mathrm{C} 3$ & $117.0(6)$ \\
$\mathrm{C} 1-\mathrm{N} 1-\mathrm{H} 1 \mathrm{~N} 1$ & $118.32(4)$ & $\mathrm{C} 1-\mathrm{C} 2-\mathrm{H} 1 \mathrm{C} 2$ & $124.4(6)$ \\
$\mathrm{C} 1-\mathrm{N} 2-\mathrm{H} 1 \mathrm{~N} 2$ & $122.5(10)$ & $\mathrm{C} 3-\mathrm{C} 2-\mathrm{H} 1 \mathrm{C} 2$ & $121.75(9)$ \\
$\mathrm{C} 1-\mathrm{N} 2-\mathrm{H} 2 \mathrm{~N} 2$ & $117.2(9)$ & $\mathrm{C} 2-\mathrm{C} 3-\mathrm{C} 2$ & $119.12(5)$ \\
$\mathrm{H} 1 \mathrm{~N} 2-\mathrm{N} 2-\mathrm{H} 2 \mathrm{~N} 2$ & $109.3(13)$ & $\mathrm{C} 2-\mathrm{C} 3-\mathrm{H} 1 \mathrm{C} 3$ & $119.12(5)$ \\
$\mathrm{N} 1-\mathrm{C} 1-\mathrm{N} 2$ & $117.81(7)$ & $\mathrm{C} 2-\mathrm{C} 3-\mathrm{H} 1 \mathrm{C} 3$ & \\
$\mathrm{~N} 1-\mathrm{C} 1-\mathrm{C} 2$ & $118.83(6)$ & & \\
\hline
\end{tabular}

Hydrogen-bond geometry $\left(\AA,{ }^{\circ}\right)$

\begin{tabular}{lllll}
\hline$D-\mathrm{H} \cdots A$ & $D-\mathrm{H}$ & $\mathrm{H} \cdots A$ & $D \cdots A$ & $D-\mathrm{H} \cdots A$ \\
\hline $\mathrm{N} 1-\mathrm{H} 1 N 1 \cdots \mathrm{C} 11$ & $0.90(2)$ & $2.18(2)$ & $3.0790(11)$ & $175.6(19)$
\end{tabular}


$\mathrm{N} 2-\mathrm{H} 2 \mathrm{~N} 2 \cdots \mathrm{C} 11^{\mathrm{i}}$

$\mathrm{N} 2-\mathrm{H} 1 N 2 \cdots \mathrm{C} 11^{\mathrm{ii}}$

Symmetry codes: (i) $x+1 / 2, y+1 / 2, z$; (ii) $-x+1 / 2,-y+1 / 2,-z$

\begin{abstract}
$0.833(13)$
\end{abstract}
$0.875(13)$
$2.628(13)$

$2.877(13)$
$3.4086(8)$

$3.3601(8)$
$156.5(12)$

$116.8(2)$ 